Meta

Journal des tradlucteurs

Translators' Journal

\title{
Histoire de la terminologie au Canada et au Québec
}

\section{Nada Kerpan}

Volume 22, numéro 1, mars 1977

Histoire de la traduction au Canada

URI : https://id.erudit.org/iderudit/003306ar

DOI : https://doi.org/10.7202/003306ar

Aller au sommaire du numéro

Éditeur(s)

Les Presses de l'Université de Montréal

ISSN

0026-0452 (imprimé)

1492-1421 (numérique)

Découvrir la revue

Citer cet article

Kerpan, N. (1977). Histoire de la terminologie au Canada et au Québec. Meta,

22(1), 45-53. https://doi.org/10.7202/003306ar d'utilisation que vous pouvez consulter en ligne.

https://apropos.erudit.org/fr/usagers/politique-dutilisation/ 


\section{Histoire de la terminologie au Canada et au Québec}

\section{ORIGINE}

La terminologie existe aujourd'hui, au Canada et au Québec, en tant que discipline et activité autonome. Non seulement s'exerce-t-elle largement et dans tout secteur, mais elle possède également ses lois et règles propres. Il existe maintenant une théorie dans ce domaine ; on assiste à une pratique terminologique poussée tant dans l'administration publique que dans l'entreprise privée ; et principes et pratique sont enseignés dans les universités et par les associations professionnelles de traducteurs.

$\mathrm{Si}$, au Canada et au Québec, la terminologie a maintenant ses titres de noblesse, elle le doit, d'une part, à une lente et longue évolution vers ce qu'il convient de nommer la différenciation ou l'autonomie et, d'autre part, à des situations linguistiques nouvelles. La promulgation du bilinguisme dans les institutions fédérales (1970) et la politique de francisation au Québec, amorcée depuis quelques années, sont, à n'en pas douter, les instruments qui ont consacré la nécessité d'une action terminologique vaste et soutenue. Le passage de l'unilinguisme anglais à un usage parallèle ou fonctionnel du français a en effet provoqué un immense appel de terminologie.

Cette activité terminologique intense n'a pas pris forme que récemment. Depuis qu'il y a contact entre les deux langues, depuis qu'il y a traduction au Canada et au Québec, il y a nécessairement terminologie. C'est, en effet, par la traduction que se sont manifestés et que continuent de se manifester, pour une très large part encore, les besoins terminologiques. D'ailleurs, la terminologie actuelle est généralement bilingue ou - pour mieux la nommer - comparée, et cette caractéristique se maintiendra vraisemblablement étant donné le prestige, l'importance et l'évolution des techniques et de la langue américaines.

Si la terminologie a su se préciser par rapport à la lexicographie, elle a également dû se définir par rapport à la traduction, exercée qu'elle l'était depuis les débuts par les traducteurs. Dans le contexte actuel, où le traducteur du Canada et du Québec est le grand artisan de la langue sur son territoire et où la demande de traduction est formidable, la terminologie voisine et voisinera encore avec l'activité de traduction; mais elle exige désormais du traducteur et du praticien une démarche propre et spécifique. 


\section{EVOLUTION}

« En Ontario existait depuis 1920 l'Association technologique de langue française d'Ottawa, ancêtre de l'ATIO, à qui l'on doit la publication de glossaires. Doyenne des groupements professionnels de traduction, cette association a donc été aussi ie premier organisme canadien à offrir aux traducteurs les fruits d'une recherche terminologique ${ }^{1}$.

Cependant, la formation du Bureau des traductions au Secrétariat d'État à Ottawa, en 1935, entraîne une activité terminologique, le plus souvent d'ordre ponctuel parce que nécessitée par les travaux de traduction divers. Cette activité n'est, en fait, régie par aucune règle; elle est laissée à l'initiative, à la curiosité, au talent du traducteur, et au hasard des textes de traduction. Ainsi, bon nombre de « fichiers individuels » se constituent - produit soit de recherches dans des lexiques existants ou dans une documentation française en général réduite, de lectures variées, ou du processus même de traduction.

En 1945, est mis au point par Pierre Daviault et publié sous la direction du chef d'état-major général, à Ottawa, un volumineux Dictionnaire militaire anglaisfrançais et français-anglais.

Par ailleurs, ce sont les fichiers personnels qui, en 1954, permettent la publication de 25 Bulletins de terminologie; y sont traités, l'administration pénitentiaire, le droit d'auteur, la terminologie des plantes particulières au Canada, la pêche commerciale et sportive, la psychiatrie, etc., ou encore des expressions diverses tels, par exemple, «parking meter, tenders called, picture window, scientist ». Au cours des années suivantes, les domaines les plus divers continuent d'être abordés dans ces publications, dont le nombre s'établit à près de 100 en 1960.

C'est encore et aussi un fichier personnel volumineux - constitué par l'intermédiaire de travaux de traduction, par des recherches, des consultations et des lectures multiples - qui produit des ouvrages tels « L'expression juste en traduction » et «Questions de langage » signés Pierre Daviault et regroupés en 1961 en Langage et traduction. L'auteur y expose une terminologie des plus variée, en traite en comparatiste et s'y fait, le cas échéant, didactique.

Durant cette même période s'élabore également un autre fichier personnel qui se traduira plus tard en un important bulletin de terminologie intitule Vocabulaire général anglais-français, d'Hector Carbonneau. Comme, dans le cas précédent, il ne s'agit point de terminologie thématique ; les termes les plus divers s'y trouvent réunis, termes relevés par l'auteur au cours de lectures, de recherches, de travaux de traduction ou de révision, ou encore pour lesquels ce dernier a do lui-même fournir des équivalents. L'ouvrage est destiné aux traducteurs, notamment aux traducteurs généralistes.

Une préoccupation terminologique existe donc au Bureau fédéral de traduction, mais il n'y a point de méthodologie établie ni de vaste objectif terminologique.

1. Paul A. Horguelin, * Historique de la recherche et de la diffusion terminologiques au Canada ", Compte rendu : Colloque sur la terminologie appliquée à la traduction, ATIO, Ottawa, septembre 1974 , p. 11. 
Les termes anglais proviennent des travaux de traduction et la terminologie française retourne au service du traducteur. Quoiqu'un Service de terminologie existe au bureau même du surintendant de la traduction, il ne compte jusqu'en 1960 qu'une seule personne et l'activité n'y est pas rigoureusement organisée. En effet, le traducteur-terminologue $y$ revoit la matière terminologique que communique la division de traduction d'un ministère, travaille à quelque sujet de son choix, ou encore fait de la recherche ponctuelle sans toutefois disposer de documentation suftisante. La publication des bulletins se poursuit, mais ce n'est qu'au début des années 60 que les autorités songent à une expansion du centre. Le Centre de terminologie aura désormais pour mission de stimuler la rédaction scientifique des fiches; regrouper le fruit des recherches terminologiques faites dans les services de traduction des divers ministères; effectuer de la recherche systématique à même les lexiques existants ou des ouvrages techniques unilingues; publier lexiques, bulletins et instructions terminologiques; assurer la consultation terminologique ; et constituer un service de documentation. En 1963, le Centre ne compte encore que deux terminologues et, au début des années 70 , le personnel en est encore peu nombreux. Dans l'intervalle, cependant, l'on continue de publier des bulletins et des instructions et, en 1968, commence à paraître l'Actualité terminologique, publication mensuelle destinée aux traducteurs et aux rédacteurs et dont les préoccupations seront tout autant linguistiques que terminologiques. En effet, les premiers numéros témoignent déjà et fidèlement de cet objectif : on y réfléchit sur la langue technique française; on y analyse des termes divers ; on y signale de la documentation; et on y décrit une méthode de recherche terminologique en fonction de la traduction.

Par ailleurs, des lexiques bilingues divers sont publiés par certains ministères ou organismes fédéraux, ou par des particuliers. Parce qu'en sont trop souvent absentes méthodologie et rigueur, la valeur en est discutable et démontre la nécessité d'un organisme de normalisation des travaux de terminologie.

Au Canada français, la terminologie trouve ses racines dans nombre de travaux lexicographiques ou normatifs. Ainsi, à la fin du $19^{\circ}$ siècle, paraissent le Glossaire franco-canadien et Vocabulaire de locutions vicieuses usitées au Canada, d'Oscar Dunn et le Dictionnaire canadien-français ou Lexique-Glossaire des mots, expressions et locutions ne se trouvant pas dans les dictionnaires courants et dont l'usage appartient surtout aux Canadiens français, de Sylvia Clapin. En 1902, est fondée la Société du parler français au Canada et le début du siècle est marqué par l'œuvre didactique de l'abbé Étienne Blanchard, d'Adjutor Rivard, de Léon Lorrain... Du premier auteur, paraissent le Manuel du bon parler ou Stylistique canadienne; le Dictionnaire du bon langage (1919) qui comprend, entre autres sujets, les termes de l'automobilisme, de la radiophonie, du tennis et de divers jeux et que l'on dit, à l'époque, \& excellent pour les traducteurs $\$$; le Bon Français en affaires; 2000 mots bilingues par l'image. En 1930, est publié le Glossaire du parler français au Canada et, durant cette première moitié du siècle, se poursuit patiemment une cuvre à la fois lexicographique, corrective et voire même terminologique. C'est ainsi que l'on retrouve, par exemple, chez l'auteur du Dictionnaire général de la langue au Canada (1954), Louis-Alexandre Bélisle, une activité 
et de traducteur et de terminologue : traduction de manuels techniques, enseignement du français des affaires et de la technique des marchés mobiliers.

En 1957, s'ajoutent les Bulletins de linguistique de l'Académie canadiennefrançaise, qui paraîtront jusqu'en 1962. "Rédigés, comme l'indique la première livraison, d'après les mises au point les plus récentes des linguistes, des savants, des techniciens de France et du Canada », ces bulletins traitent de questions de vocabulaire, de grammaire ainsi que de l'actualité linguistique. On y étudie, par exemple et très souvent, les anglicismes : développement, pipe-line, parking, dispatch, etc.

Bref, la réflexion sur les mots et la langue semble être de très longue tradition au Canada français, et sans hiatus. Toutefois, cette réflexion et action ne demeurera plus, dans les années 60 , le fait de quelques individus ou organisations et dépassera l'aspect correctif ou lexicographique de la langue. Elle gagnera divers milieux, touchera les domaines et les techniques les plus nouvelles, s'exercera de façon plus spécifique, concrète et officielle, s'acheminant discrètement vers la différenciation.

C'est ainsi que « la création du Comité de linguistique de Radio-Canada, en mars 1960 , va... marquer une étape importante... Å ses débuts, le Comité a une action purement corrective et limitée au personnel de Radio-Canada. Mais bientôt, par la publication du bulletin C'est-à-dire et des « fiches » qui l'accompagnent, il déborde la terminologie de la radio-télévision et, audace rare à l'époque, n'hésite pas à créer des néologismes... ${ }^{2}$ " de facture canadienne.

En 1961, est créé — fait capital dans l'histoire de la terminologie l'Office de la langue française ; il a pour vocation la promotion intégrale du français. \&Il serait mauvais de fonder, écrit le ministre des Affaires culturelles de l'époque, l'œuvre de l'Office sur une simple défense de la langue française. Des organismes spécialisés, explique-t-il, sont nécessaires pour fournir aux grandes langues de civilisation les moyens d'exprimer l'extraordinaire essor scientifique et technique... ${ }^{3} \gg$ Normative à ses débuts, l'activité de l'Office devient vite terminologique. Dès 1962, paraît le bulletin Mieux dire, qui reproduit les fiches et travaux de divers organismes français, présente les lexiques établis par l'Office même, et aborde la correction du langage. Commencent ensuite à paraître les Cahiers, dont les premiers exposent une «Norme du français écrit et parlé au Québec ( (1965), les «Canadianismes de bon aloi (1969), "Quel français devons-nous enseigner? \$(1970). S'y ajoutent, dans l'intervalle, des vocabulaires traitant d'assurances sociales, d'assurance-vie, de radio et télévision. Sont également organisés des colloques : en 1966, un colloque sur la normalisation et la diffusion des terminologies techniques et scientifiques et, en 1968, un colloque sur la traduction auquel on recommande l'établissement d'un centre de documentation automatique en vue des besoins terminologiques en traduction ${ }^{4}$. En 1969 , est conçu un programme de traitement électronique de l'information termi-

2. Paul A. Horguelin, op. cit., p. 12.

3. Mieux dire, bulletin de linguistique de l'Office de la langue française, no 1 , Montréal, octobre 1962, p. 1.

4. Paul A. Horguelin, op. cit., p. 12. 
nologique, qui sera réalisé avec le concours du Centre de documentation de 1'Université Laval. Et voilà ainsi atteine une nouvelle étape dans la structuration de la terminologie.

Par ailleurs, la création de services de traduction dans les entreprises de Montréal, ou l'expansion des quelques services existants, provoquent un accroissement de l'activité terminologique. Se constituent des fichiers qui consignent le fruit de recherches thématiques mais plus souvent de recherches nécessitées par les textes de traduction, et paraissent nombre de lexiques bilingues. $\grave{A}$ Bell Canada, par exemple, est établi en 1965 un fichier groupant plus de 1200 désignations des postes cadres de l'entreprise, et sont publiés, à partir du fichier général et pour les besoins internes, divers glossaires techniques. À la CIL est diffusée en 1965 une centaine de fiches techniques. A l'Hydro-Québec paraissent en 1967 un Dictionnaire électrotechnique ainsi qu'un Vocabulaire nucléaire. Domtar, qui avait publié, dès 1948 , le Dictionnaire de la terminologie des industries $d u$ papier, poursuit son activité terminologique. Les Chemins de fer nationaux sont, pour leur part, les premiers à disposer d'un service de terminologie autonorne ; formé en 1966, à la fois pour les besoins du personnel de l'entreprise et du service de traduction, le service publie, dès 1968, les Vocabs, feuillets terminologiques illustrés d'une haute qualité technique et linguistique. Toutefois, si l'activité terminologique décuple dans l'entreprise, elle ne sait toujours s'effectuer avec rigueur. Soumise aux contraintes des délais courts de traduction et réalisée par des sujets exclusivement orientés vers la traduction ou non initiés à la démarche terminologique, elle devient trop souvent - comme au Bureau fédéral de traduction - un pur relevé de termes tirés de dictionnaires bilingues ou produits par le processus de traduction. On ignore, ou presque, la technique du dépouillement parallèle d'ouvrages techniques, ou encore on ne dispose d'aucune documentation du genre. C'est ainsi que paraîtront, et seront diffusés dans le public, des lexiques d'une valeur douteuse, où des termes maison voisineront avec des expressions traduites, voire même avec des anglicismes.

Les années 60 connaissent donc une effervescence terminologique sans précédent. En 1963, par exemple, l'Accounting Terminology de l'Institut canadien des comptables agréés publié en 1957, à Toronto, s'accompagne désormais des "Termes comptables », lexique bilingue ; en 1965, est formé le Comité de terminologie française de l'Ordre des comptables agréés du Québec, dont le mandat est de " travailler à répandre le bon usage en matière de terminologie comptable française parmi les membres de l'Institut, les étudiants et le large public du monde des affaires », et dont l'objectif immédiat est une publication périodique Terminologie comptable ${ }^{5}$. En 1965, la Société des traducteurs et interprètes du Canada organise, à Stanley House (en Gaspésie), un colloque de traducteurs et de linguistes dont l'une des conclusions porte sur la nécessité, pour le traducteur, de dépouiller les articles et les ouvrages se rapportant à la matière des textes qu'il traduit et, pour un bureau de traduction, de disposer d'un personnel faisant uniquement du dépouillement. Jean Darbelnet publie, dans le numéro XXVI (1965) de Culture,

5. Terminologie comptable, Comité de terminologie française, Institut des comptables agréés du Québec, no 1 , août 1966, p. 1 , 
un article intitulé \& Pour une meilleure coordination de la recherche et de la documentation en terminologie ». Meta, journal des traducteurs, accueille dans ses pages études terminologiques et vocabulaires. Paraît, en 1968, un Vocabulaire de l'éducation au Québec, établi par le ministère de l'Éducation ; un peu plus tard, par exemple, un Dictionnaire bilingue de la prévention des incendies, du ministère des Affaires municipales.

\section{ANNÉES 1970}

La décennie débute dans une quasi-fébrilité terminologique, la terminologie s'exerçant à peu près partout, cherchant à s'organiser rationnellement et s'érigeant en théorie.

En octobre 1970, un nouvel événement capital se produit : la création de la Banque de terminologie de l'Université de Montréal. L'idée d'une banque de mots avait été formulée au colloque de Stanley House en 1965, puis reprise à un colloque de l'Office de la langue française en 1968 ; et le projet, étudié en comité, reçoit l'appui des gouvernements du Québec et d'Ottawa, de l'Université de Montréal et d'un certain nombre d'entreprises privées, en juillet 1969. La terminologie va y être pratiquée, sans intention de normalisation, «comme discipline autonome, mais ordonnée vers la traduction, dont l'objet est l'étude descriptive du vocabulaire de spécialités en situation concrète d'emploi ${ }^{6} \$$. C'est ainsi que TERMIUM, fichier de la Banque, contient en 1975 quelque 75000 termes scientifiques et techniques, auxquels s'ajoutent des expressions semi-techniques d'usage courant. En février de la même année, s'effectue une expérience-pilote de 6 mois avec les Services linguistiques de Bell Canada, abonnement expérimental qui confirme la nécessité de fichiers automatisés tant à l'échelle du Québec, du Canada qu'au sein des entreprises mêmes. En janvier 1976, la Banque devient la propriété du Secrétariat d'État.

Outre l'œuvre de pionnier accomplie en matière d'automatisation des fichiers et l'apport considérable au fonds terminologique général ainsi qu'à la méthodologie terminologique même, la Banque a également une action marquée sur le plan pédagogique. Par les stages d'été réservés aux étudiants de traduction, elle forme à la discipline de la terminologie nombre de sujets pour qui la traduction et la terminologie deviennent deux domaines distincts parce que réclamant des démarches propres.

A Ottawa, par ailleurs, succède en 1971 au Centre de terminologie une Division de la recherche terminologique et linguistique, dont l'objectif est double : recherche et information. La division doit rassembler les termes ressortissant a des domaines lexicaux donnés; recueillir les nouveautés linguistiques; enrichir le fichier par le dépouillement de publications; diffuser le résultat au moyen de lexiques et de l'Actualité terminologique; et assurer un service de renseignements aux traducteurs. Le nombre de terminologues y augmente, le centre de documentation prend de l'ampleur, et les administrateurs songent à une banque

6. R. Dubuc et J.-Fr. Grégoire, * Banque de terminologie et traduction *, Babel, n⿳ 4, 1974, p. 181 . 
de mots pour faciliter au maximum la tâche du traducteur. En mars 1975, est créée Mission Terminologie qui dresse, entre autres programmes, le plan d'implantation d'une banque de terminologie devant satisfaire aux besoins des traducteurs et rédacteurs. En janvier 1976, a lieu une nouvelle réorganisation des services de terminologie qui consiste dans l'établissement d'une direction de la terminologie et de la documentation, subdivisée en trois directions : recherche terminologique, normalisation et liaison linguistique, documentation et exploitation. Le personnel comprend 80 terminologues décentralisés et le fonds terminologique compte 1400000 fiches provenant des diverses sections du bureau ainsi que des fichiers personnels des traducteurs - fiches devant être confiées à TERMIUM II et normalisées par étapes. En 1980, le Secrétariat aura à son service 200 terminologues.

Au Québec, la pratique de la terminologie gagne en intensité non seulement à cause de la Banque de terminologie de l'Université de Montréal, mais encore en raison de l'action soutenue et globale de l'Office de la langue française et de l'activité terminologique des entreprises. Celles-ci, de plus en plus nombreuses à posséder un service de traduction, sont inévitablement amenées à un exercice continu et plus poussé de la terminologie. Les fichiers d'entreprise se constituent; paraissent soudain nombre de lexiques, diffusés soit au sein de l'entreprise soit dans le public; et commencent à paraître des bulletins linguistiques, tel l'intéressant Langue-Information de la CIL en 1971. Cependant, comme dans la décennie précédente, la terminologie y est trop souvent tirée des divers dictionnaires bilingues sans évaluation critique des ouvrages consultés; enfin, elle est parfois même recueillie ou établie par des individus tout à fait étrangers à la terminologie, à la traduction ou encore à la rédaction. Mais, peu à peu se créent des postes de terminologues et des services de terminologie. En 1974, est rédigée par un groupe de l'Université du Québec à Montréal, et avec le concours de quelques terminologues d'entreprise et d'un terminologue de TERMIUM, une première analyse des tâches du terminologue. En 1976, on compte encore peu de terminologues en titre ou en exercice mais, de plus en plus, les employeurs exigent du traducteur la fonction de terminologue - comme en témoignent les offres d'emplois actuelles. Par ailleurs, des Comités de terminologie se forment spontanément en électrotechnique, en assurances, en télécommunications, etc.

L'Office de la langue française, pour sa part, reçoit un mandat élargi en 1969. Non seulement doit-il continuer à corriger et à enrichir la langue parlée et écrite au Québec, mais encore il doit favoriser l'implantation du français dans les entreprises. Aussi met-il sur pied un service composé de linguistes et de traducteurs dont la tâche est de dresser des vocabulaires, voire même d'étudier les vocabulaires techniques en situation industrielle.

En juillet 1974, le mandat de l'Office - qui devient la Régie de la langue française - est encore plus vaste : la mise en application de la Loi sur la langue officielle. La loi, qui prévoit le développement et la coordination de la recherche en matière linguistique, la création de commissions de terminologie, la mise en cuvre de programmes de francisation d'entreprises, et la normalisation du voca- 
bulaire utilisé au Québec, consacre le bcsoin d'une intensification de l'activité terminologique.

Déjà, l'Office avait mis au point un nombre considérable de vocabulaires et cette activité, poursuivie sans relâche, se traduit aujourd'hui par des publications terminologiques touchant tout autant l'alimentation, les assurances, l'automobile, le conmerce, la gestion, la sécurité, le sport, les métiers, les secteurs industriels et techniques que la néologie et la langue proprement dite. Soucieuse d'efficacité et de normalisation, la Régie organise, dans le cadre de la coopération francoquébécoise, des missions documentaires et terminologiques qu'elle confie à ses propres terminologues ou encore aux entreprises. Soucieuse de coordination, elle réalise - pour faire suite au vœu du colloque de Baie Saint-Paul - un inventaire annuel des travaux de terminologie au Québec, inventaire qu'elle étend maintenant à la France.

Parallèlement à ces travaux de terminologie, la Régie poursuit la réalisation de la Banque de terminologie du Québec, mise en chantier en 1969 et devant être accessible au public en 1977. Aujourd'hui, cette Banque comprend : TERMINOQ 1, fichier automatisé de terminologie contenant plus de 160000 fiches, et TERMINOQ 2, inventaire des ouvrages et travaux de terminologie.

Cependant, la contribution de la Régie à la terminologie dépasse la simple production de mots ou de lexiques pour toucher aussi la théorie. En effet, au cours des années, se poursuit une réflexion qui porte sur la discipline, sur sa fonction et sur sa méthodologie - réflexion qui se manifeste par des colloques ou des publications sur le sujet. Plusieurs colloques internationaux se tiennent au Québec : celui de 1972 permet de fixer les données minimales de la fiche terminologique ; celui de 1973 aborde la normalisation ; celui de 1974 traite de l'aménagement de la néologie; celui de 1975 est un essai de définition de la terminologie. Dans l'intervalle, est publié un Guide de travail en terminologie (cahier $n^{\circ} 20$ ), dont une édition révisée est prévue pour le début de 1977 ; et suivra un Manuel de terminologie vers la fin de la même année.

Aujourd'hui, la direction de la terminologie de la Régie comprend 3 services - les groupes de la recherche terminologique, de la Banque de terminologie et des linguistes-conseils, soit près de 150 terminologues.

A l'exercice de la terminologie par le Secrétariat d'État à Ottawa, par la Régie de la langue française, par les entreprises et même les particuliers, et à l'élaboration d'une théorie de la terminologie, s'ajoute l'enseignement de cette discipline. L'Université de Montréal est première à l'intégrer au programme des cours de traduction et à accepter des travaux de terminologie comme thèses ou mémoires ; l'Université Laval décide, aussi d'introduire la terminologie aux cours de traduction et de l'enseigner magistralement; l'Université du Québec à TroisRivières étudie, en 1974, l'opportunité d'instituer une maîtrise en terminologie ; l'Université d'Ottawa, pour sa part, assure, depuis 1973, un cours d'initiation à la recherche terminologique. Les associations professionnelles de traducteurs dispensent, de leur côté, un enseignement pratique - telles la STQ et l'ATIO. La Banque de terminologie de l'Université de Montréal, comme il était précédem- 
ment, fait aussi ouvre didactique et la Régie, pour sa part, songe à une action pédagogique dans un proche avenir.

La présente décennie assiste donc à un mouvement terminologique général. En 1973, on tente de créer - avec l'aide du Conseil des sciences du Canada un Comité international de terminologie scientifique et technique (CITEST). D'autres colloques ont lieu : en septembre 1974, l'ATIO organise un colloque sur la terminologie appliquée à la traduction et, en novembre, reprend à son congrès annuel le thème de la diffusion; en janvier 1976, se tient, sous les auspices de la Régie une première rencontre des terminologues et traducteurs-terminologues du Québec; en février 1976, a lieu, à l'initiative du Secrétariat d'État d'Ottawa, un colloque canadien sur les fondements d'une méthodologie générale de la recherche et de la normalisation en terminologie et en documentation ; en novembre 1976, est organisée une deuxième rencontre des terminologues et traducteurs-terminologues du Québec, rencontre qui porte sur la structure des comités de terminologie. Par ailleurs, des comités de terminologie intraentreprises, interentreprises ou gouvernementaux continuent de se former en assurances, électrotechnique, télécommunications, pétroles, etc., ou encore le Comité de terminologie de la Régie de l'assurance-maladie, par exemple. Des dictionnaires automatisés sont élaborés dans les universités : en droit, DATUM à l'Université de Montréal et JURIVOC à Ottawa; l'Université de Sherbrooke travaille à un dictionnaire médical bilingue. Enfin, le Secrétariat d'État, la Régie et l'entreprise participent aux comités consultatifs canadiens de l'ISO.

En 1976, la terminologie s'exerce sur tous les fronts au Québec et au Canada, et a même un rayonnement international. Il ne lui reste plus qu'à poursuivre son effort de coordination et à en arriver à une méthodologie commune pour que puissent être satisfaits le mieux et le plus vite possible les besoins forrnidables actuels.

NADA KERPAN 